\title{
Incorporating psycho-physical mapping into random regret choice models: model specifications and empirical performance assessments
}

\author{
Sunghoon Jang ${ }^{1} \cdot$ Soora Rasouli $^{1} \cdot$ Harry Timmermans ${ }^{1}$
}

(C) The Author(s) 2016. This article is published with open access at Springerlink.com

\begin{abstract}
Recently, regret-based choice models have been introduced in the travel behavior research community as an alternative to expected/random utility models. The fundamental proposition of regret theory is that individuals minimize the amount of regret they (are expected to) experience when choosing among choice alternatives. In this context, regret is defined as a function of attribute differences between the considered choice alternative and one or more foregone choice alternatives in an individual's choice set. This definition of regret as a function of physical attributes of the choice alternatives implies that current regret-based choice models do not account for the perception of attributes, which is likely related to their magnitude. Therefore, in this paper, we propose and empirically test such an elaboration of the basic regret-minimization models. The current paper sets out to formulate random regret minimization models that incorporate a nonlinear representation of the perception of attribute levels. Inspired by long-standing research on psycho-physical measurement, it is assumed that the perception of stimuli (attributes) is proportional to their magnitude. To allow for slight deviations from this representation, a more general non-linear psycho-physical representation of the relationship between attribute levels and their perception is also tested. The suggested models are tested using two data sets, one data set concerned the stated choice of shopping centre, the other concerned revealed preference of mode choice. The two newly formulated regret models are compared against each other and against their original random regret minimization base specifications. In addition to comparing the predictive performance of these model specifications, validation tests are conducted. In each case study, the newly
\end{abstract}

Sunghoon Jang

s.jang@tue.nl

Soora Rasouli

s.rasouli@tue.nl

Harry Timmermans

h.j.p.timmermans@tue.nl

1 Urban Planning Group, Department of Urban Science and Systems, Eindhoven University of Technology, P. O. Box 513, 5600MB Eindhoven, The Netherlands 
suggested regret models, incorporating a non-linear representation of perception, achieve significant improvements in goodness-of-fit over the original regret formulations. The results of the K-fold validation tests provide further support to this finding.

Keywords Regret-based choice models - Psycho-physical relationship - Cross-validation test $\cdot$ Stated choice experiment $\cdot$ Revealed choice experiment

\section{Motivation}

In search of alternative behavioral foundations of choice models, the concept of regret has recently attracted the attention of several scholars in transportation research (Chorus et al. 2008a, b; Chorus 2010; De Moraes Ramos et al. 2011; Hess et al. 2012; Chorus 2012a, 2012b; Kaplan and Prato 2012; Chorus and Bierlaire 2013; Chorus et al. 2013b; Hensher et al. 2013; Boeri and Masiero 2014; Chorus 2014a, b; Prato 2014; Rasouli and Timmermans 2015a). Regret relates to an affective state of mind that individuals may experience or anticipate once they realize that one or more of the non-chosen choice alternatives outperform the chosen alternative on one or more attributes of interest. Regretbased models assume that individuals minimize regret rather than maximize utility when choosing among choice alternatives. The principle of regret minimization may signal bounded rationality in the sense that emotions are assumed to play a role in the decisionmaking process and that individuals tend to avoid extremes in their decision processes (Rasouli and Timmermans 2015b).

Chorus et al. (2008a) introduced regret-based models into transportation research. They provided an econometric framework extending seminal work (Bell 1982; Loomes and Sugden 1982, 1983, 1987; Quiggin 1994), from binary to multinomial and from single to multi-attribute choices. Originally, they defined regret as a linear function of negative attribute differences between the considered alternative and the best non-chosen alternative. Later, Chorus (2010) suggested replacing this discontinuous function with a continuous logarithmic approximation, primarily for technical reasons. Very recently, Chorus (2014c) introduced the generalized regret model, but we do not include it in the present study because it deviates too much from the pure notion of regret.

This fundamental work has led to an upsurge of applications of regret-based models, and extensions of the model under both certain conditions (Chorus et al. 2008a, b; Chorus and de Jong 2011; Chorus 2012b; Kaplan and Prato 2012; Beck et al. 2013; Chorus et al. 2013; Chorus 2014a; Hensher et al. 2013; Boeri and Masiero 2014, Prato 2014), and uncertain conditions (Chorus et al. 2008a; De Moraes Ramos et al. 2011; Chorus 2014b). Regret-based models seem to be more important when respondents are less familiar with the choice situation (probably because they have less defined preferences) (Boeri et al. 2014). Applications cannot ony be found in transportation research area but also in environmental studies (Thiene et al. 2012) and health economics (de Bekker-Grob and Chorus 2013).

All these regret-based models have in common that regret is a function of attributes differences of the choice alternatives. That is, the physical measurements of attributes are used in the regret function to measure the amount of regret, assuming that regret is based on these differences. In reality, however, regret may be based on the perception of physical attribute differences. Psycho-physical research, although focusing on different kind of stimuli such as noise, has provided a substantial amount of evidence that the relationship between physical attribute values and perceived attribute values is non-linear. If these 
findings would equally apply to travel-related attributes, current regret-based models would wrongly represent the mental representation of physical attributes levels that are subsequently used to arrive at a choice. All current regret-based choice models implicitly or explicitly ignore the perception of attributes. Compared to utility-based models of transport-related decisions, ignoring perception may have more dramatic repercussions, because regret-based models are based on attribute differences. It is assumed that the same absolute attribute difference is perceived independently of the overall attribute range and the absolute value of attribute itself. Thus, incorporating an improved representation of the perception of attributes into regret-based models may enhance their predictive success.

In this paper, we therefore propose and empirically test such an elaboration of the basic model specifications. In order to investigate this issue, the current paper sets out to formulate elaborations of the basic random regret minimization models and incorporates a non-linear representation of the perception of attributes. Inspired by research on psychophysical measurement, it is assumed that the perception of stimuli (attributes) is proportional to their absolute values. To allow for slight deviations from this representation, a more general non-linear representation is also formulated and tested. Two data sets (one on stated choice, the other on revealed choices) are used to estimate and assess the proposed models. These newly formulated regret models will be compared against each other and against the original random regret minimization models. In addition to comparing their predictive performance, K-fold validation tests will be conducted.

The remainder of the paper is organized as follows. We will first summarize current regret-based models as they have been formulated in transportation research. Next, we will outline the new regret models that incorporate the non-linear psycho-physical relationships. Then, we will discuss the estimation and validation results for two case studies, respectively pertaining to choice of shopping center and transportation mode choice. The paper is completed with a discussion of the results and avenues of future research.

\section{Conceptual framework}

The urban-transportation system can be described in terms of a set of $i=1,2, \ldots, I \in C_{n}$ choice alternatives, characterized by a non-empty set of $K_{i}$ attributes, defining choice set $C$ of individual $n$. The set of attributes may be identical for the different choice alternatives (e.g., a set of generic attributes for shopping centres), but may also be (partly) specific for different alternatives (e.g., attributes of transport modes). Let $X_{i k}$ describe the attribute value of alternative $i$ on attribute $k$. Finally, let $Z_{n j}, j=1,2, \ldots, J$ describe characteristic $j$ of decision-maker $n=1,2, \ldots, N$.

Random utility and random regret models directly link attribute values of the choice alternatives to the choices, observed in the real world $\left\{X_{i k}\right\} \rightarrow p_{i}, i \in C$ where $p_{i}$ is the probability of choosing alternative i. In formulating an operational model, a researcher has to select the set of attributes $\left\{K_{i}\right\}$, assumed to influence the choice behaviour of interest, and specify choice set $C$, which may be individual specific or universal. If the modelling approach predicts observed probabilities as a function of the objective attribute values (revealed preference), there is no explicit attempt to further detail and breakdown the decision making process.

The choice literature, however, documents several attempts and more detailed ways to elaborate this approach. In this study, we focus our attention to the relationship between the objective values of the physical attributes and their perception by the decision maker. This 
psycho-physical relationship involves the mapping of the objective, physical space into a cognitive space, measuring individuals' perception: $\left\{X_{i k}\right\} \rightarrow\left\{x_{n i k}\right\} \forall i \in C ; \forall n ; \forall k \in K_{i}$. This elaboration would acknowledge that individuals hold partial $\left(k_{i} \subset K_{i}\right)$ and imperfect knowledge $x_{n i k} \neq X_{i k}$ about the attributes of the choice alternatives in their choice set. Moreover, individuals may not be aware of all choice alternatives $\left(C_{n} \subset C\right)$. This elaboration also contends that individuals base their decisions on their cognitive representations of reality as opposed to reality itself. The notion that knowledge is imperfect may be captured by any non-identity function, mapping objective attribute values into cognitive values: $x_{n i k}=f_{n k}\left(X_{i k}\right) \forall i \in C_{n} ; \forall n ; \forall k \in K_{n i}$ where $f_{n k}$ is any function applied to attribute $k$ by individual $n$.

The classic random regret model, suggested by Chorus et al. (2008a, b), assumes that function $f$ is an identity function, indicating that objective attribute values as opposed to cognitive or perceived values are used. The original attribute-level regret function is assumed to be equal to

$$
\varphi_{i i^{\prime} k}=\max \left[0, \beta_{k}^{\mathrm{BO}}\left(X_{i^{\prime} k}-X_{i k}\right)\right]
$$

where $\varphi_{i i^{\prime} k}$ defines the regret for chosen alternative $i$ when compared to foregone alternative $i^{\prime}$ on attribute $k$, operator. Then, the regret of chosen alternative $i$ when compared to foregone alternative $i^{\prime}$ is assumed equal to the sum of attribute-level regrets.

$$
R_{i i^{\prime}}=\sum_{k} \varphi_{i i^{\prime} k}
$$

Moreover, to generalize regret to the multi-alternatives choice situation, following Quiggin (1994) and assuming a universal choice set, the regret of chosen alternative $i$ is defined against the best foregone alternative $i$ :

$$
R_{i}^{\mathrm{BO}}=\max _{i^{\prime} \neq i \in C}\left\{R_{i i^{\prime}}\right\}
$$

(BO) stands for the "Best Only" regret based choice model. In this paper, we will refer to this model specification as the original regret model.

Because this discontinuous specification of regret leads to possible estimation problems, Chorus (2010) approximated the discontinuous attribute-level regret function by the following continuous logarithmic function:

$$
\varphi_{i i^{\prime} k}^{\mathrm{L}}=\ln \left(1+\exp \left[\beta_{k}^{\mathrm{PL}}\left(X_{i^{\prime} k}-X_{i k}\right)\right]\right)
$$

As in the original regret model, the regret associated with chosen alternative $i$ when compared to foregone alternative $i^{\prime}$ is defined as the sum of the attribute level regrets.

$$
R_{i i^{\prime}}^{\mathrm{L}}=\sum_{k} \varphi_{i i^{\prime} k}^{\mathrm{L}}
$$

In addition, he assumed that regret was not defined against the best foregone alternative, but rather against all non-chosen alternatives. That is,

$$
R_{i}^{\mathrm{PL}}=\sum_{i^{\prime} \neq i \in C} R_{i i^{\prime}}^{\mathrm{L}}
$$

PL stands for "Paired Logarithmic" regret based model. In this paper, this model specification will be called the logarithmic specification.To map regret into choice probabilities, 
both model specifications assumed that regret is a stochastic variable. Following classic discrete choice models, assuming that the random components of the regret functions are independently and identically Gumbel distributed, the assumption of regret minimization then leads to the following choice model:

$$
P_{i}=\frac{\exp \left(-R_{i}\right)}{\sum_{i^{\prime} \in C} \exp \left(-R_{i^{\prime}}\right)}
$$

where $P_{i}$ is choice probability choosing alternative $i, R_{i}$ is the regret function.

\section{New regret models}

To include perception into these base regret models, we suggest a psycho-physical relationship that can be traced back to Weber (1834). He argued that individuals perceive change in a stimulus as a constant ratio of the original stimulus. This assertion, the so called Weber's Law, indicates that as the initial stimulus gains more strength, much more strength is required for later stimuli to maintain the same perception difference.

$$
\frac{\Delta X_{k}}{X_{k}}=\mathrm{C}
$$

where $C$ is a constant (Gescheider 1997), $X_{k}$ means an initial stimuli magnitude, and $\Delta X_{k}$ means the variance of the stimuli magnitude.Regret-based choice models have been developed based on the definition that attribute-regret is proportional to attribute-differences when an alternative performs better than others. By definition, the same attributedifference generates the same regret regardless of the magnitude of the attribute.

However, considering individuals' perception in real choice situations, the same attribute-difference may be perceived smaller when the size of the attribute is larger. For instance, if someone travelled during $120 \mathrm{~min}$, he would feel a certain amount of regret if the travel time of the alternative, non-chosen route turned out to be $110 \mathrm{~min}$. However, if that person travelled for $20 \mathrm{~min}$, and the travel time of the alternative, non-chosen route would be $10 \mathrm{~min}$, would that person experience the same amount of regret? The answer to this question, according to classic regret models would be yes, because regret only depends on attribute differences, which are the same in this example. However, it is likely that the same 10 min travel time difference is perceived quite differently in these two cases.

Therefore, to avoid this inherent limitation of the current regret formulations, we assume that individuals perceive attribute differences "Based On Weber's law" (BOW). When it is applied to the original regret model, the attribute-level regret of alternative $i$ when compared to alternative $i$ ' for attribute $k$ is then defined as

$$
R_{i}^{\mathrm{BOW}}=\max _{i^{\prime} \neq i \in C}\left(\sum_{k} \max \left[0, \beta_{k}^{\mathrm{BOW}}\left(\frac{X_{i^{\prime} k}-X_{i k}}{X_{i k}}\right)\right]\right)
$$

This new regret model can describe the variance in perception with respect to the size of attribute. It implies the contention that regret is not only a function of the attribute differences of the considered alternative and the best non-chosen one but also of attribute magnitude itself. The larger the attribute size, the smaller the regret with respect to the same attribute-differences. Parameters $\beta_{k}^{\mathrm{BOW}}$ are to be estimated from observed choices. 
Likewise, the Weber's law can be easily applied to the logarithmic specification. Equation (10) for "Paired Logarithmic Weber's law" (PLW) gives the result:

$$
R_{i}^{\mathrm{PLW}}=\sum_{i^{\prime} \neq i \in C} \sum_{k} \ln \left(1+\exp \left[\beta_{k}^{\mathrm{PLW}}\left(\frac{X_{i^{\prime} k}-X_{i k}}{X_{i k}}\right)\right]\right)
$$

This model also incorporates the non-linear mapping of attribute differences into cognitive differences. Later, Guilford (1932) pointed out that the Weber's law does not hold for the perception of high intensity stimuli. Based on a series of experiments, he concluded that individuals in that case perceive change in a stimulus to be smaller, meaning that the stimulus in the denominator used as a reference should be decreased. He suggested a power coefficient in the denominator which should be less than one. The power coefficient $\vartheta$ is expected to lie between 0 and 1 .

$$
\frac{\Delta X_{k}}{\left(X_{k}\right)^{\vartheta_{k}}}=\mathrm{C}
$$

By adding the power coefficient, the suggested model is more flexible in capturing psycho-physical relationships. In this study, we will call this modification the generalized Weber's law abbreviated as (BOGW) for (Based On Generalized Weber's law. With this law, the original attribute-level regret of alternative $i$ when compared to the best alternative $i$ ' for attribute $k$ is defined as

$$
R_{i}^{\mathrm{BOGW}}=\max _{i^{\prime} \neq i \in C}\left(\sum_{k} \max \left[0, \beta_{k}^{\mathrm{BOGW}}\left(\frac{X_{i^{\prime} k}-X_{i k}}{\left(X_{i k}\right)^{\vartheta_{k}}}\right)\right]\right)
$$

Similar to the first new regret model (Eq. 10), this second regret model can also describe the variance in perception with respect to the size of the attributes of an alternative. However, the degree of variance in the denominator is smaller as captured by the power function in the denominator. Parameters $\beta_{k}^{\mathrm{BOGW}}$ should be estimated from observed choices.

Likewise, the modification can be easily applied to the attribute-level regret of the logarithmic specification, as shown in Eq. 13.

$$
R_{i}^{\mathrm{PLGW}}=\sum_{i^{\prime} \neq i \in C} \sum_{k} \ln \left(1+\exp \left[\beta_{k}^{\mathrm{PLGW}}\left(\frac{X_{i^{\prime} k}-X_{i k}}{\left(X_{i k}\right)^{\vartheta_{k}}}\right)\right]\right)
$$

Note that if the power coefficient $\vartheta_{k}$ is estimated to be equal to zero, or not statistically significant from zero, the new regret models expressed in Eqs. 12 and 13 become the original formulations (Chorus et al. 2008a, b; Chorus 2010). On the other hand, if it is equal to one, it means that regret is generated based on Weber's law (Weber 1834).

Both new regret models represent the psycho-physical relationship by dividing attribute differences by the size of attribute (moderated by some power coefficient). Therefore, individuals perceive different degrees of regret of each alternative, even for same attribute level difference, depending on the size of attribute of the chosen alternative.

To illustrate these models, assume two alternative routes (route 1 and route 2) that define the available choice alternatives between an origin and a destination, characterized by travel time only. We formulate five scenarios with a constant travel time difference between the two modes, assuming the route 1 is the faster route in all scenarios. The traveler values travel time (differences) as $-0.5 / \mathrm{min}$, and the power coefficient is assumed 
Table 1 Change in choice probability by size of attribute in three regret-based models

\begin{tabular}{llllllll}
\hline \multicolumn{7}{l}{ Travel time (min) } & \multicolumn{7}{l}{ Probability $(\%)$} \\
\hline Car & Bus & $\mathrm{R}_{\text {car }}^{\mathrm{a}}$ & $\mathrm{R}_{\text {bus }}$ & $\mathrm{WR}_{\text {car }}^{\mathrm{b}}$ & $\mathrm{WR}_{\text {bus }}$ & $\mathrm{GWR}_{\text {car }}^{\mathrm{c}}$ & $\mathrm{GWR}_{\text {bus }}$ \\
10 & 20 & 99.33 & 0.67 & 56.22 & 43.78 & 64.89 & 35.11 \\
30 & 40 & 99.33 & 0.67 & 53.12 & 46.88 & 59.34 & 40.66 \\
50 & 60 & 99.33 & 0.67 & 52.08 & 47.92 & 57.07 & 42.93 \\
70 & 80 & 99.33 & 0.67 & 51.56 & 48.44 & 55.79 & 44.21 \\
90 & 100 & 99.33 & 0.67 & 51.25 & 48.75 & 54.96 & 45.04 \\
\hline
\end{tabular}

\footnotetext{
${ }^{\text {a }}$ Original regret

b Variant of original regret based on Weber's law

c Variant of original regret based on generalized Weber's law
}

to be equal to 0.7 . Intuitively, it is likely that most individuals will choose the route 1 due to the shorter travel time when the size of the attribute is small. However, when the size of the attribute increases, the shares of the route 1 and route 2 becomes more similar. Sheffi (1985) pointed out that this expected behavior stems from the variance in perception of travel time differences as a function of absolute travel time.

Table 1 shows how choice behavior is predicted to differ for the three types of regret models (the two newly suggested regret models, and the original regret model) by size of attribute. The probability of choosing the car is always higher, but differences in choice probability between the two alternatives are not the same for the models. Like the multinomial logit model, the original regret formulation does not consider the absolute size of travel time because regret is defined only by attribute-differences between alternatives. Therefore, most individuals are predicted to choose the car in all these scenarios with the same probability, regardless of the size of the attribute. In contrast, the new regret models suggested in this paper do consider the size of the travel time attribute. When applying the original Weber's law to the specification of regret, the choice probabilities of the two alternatives become closer as the size of the attribute (travel time) become larger. In case of the generalized Weber's law, the effect of perception on choice probabilities is moderated, because the value of the power coefficient decreases the value of the denominator.

\section{Case studies}

To examine whether indeed the inclusion of the psycho-physical relationship into the original model specifications improves the predictive ability of the regret choice models, two data sets were used. The first data set concerns a stated choice experiment about the choice of shopping center. The second data set is concerned with the choice of transportation mode in a revealed preference setting. For each data set, three analyses were conducted: (i) a comparison of the goodness-of-fit of the model specification based on Weber's law versus the original two model specifications (the best only and the logarithmic pairwise comparisons models); (ii) a comparison of the goodness-of-fit of the model specification based on the generalized Weber's law versus the original two model specifications (the best only and the logarithmic pairwise comparisons models); (iii) as a byproduct a comparison of the goodness-of-fit of the original regret model versus the logarithmic specification. Technically, the latter comparison is a replication of an earlier study 
(Rasouli and Timmermans 2015a), using new data sets. In addition to the comparison of model performance, a K-fold cross-validation test was conducted for each case.

Likelihood values were calculated to compare the goodness-of-fit of the models. Because we need to compare non-nested models with different numbers of estimated parameters (specification based on the generalized Weber's law), we applied Ben-Akiva and Swait's test (1986) for non-nested choice models. ${ }^{1}$ Matlab R 2015a was used to develop the code to estimate the various models.

The K-fold cross-validation implies that the data were split into K subsets. K-1 subsets were used to estimate the model, and the remaining subset was used to test the model. This process is repeated $\mathrm{K}$ times since each subset should be used as a test set once. The 2norms statistic, which quantifies the distance between predicted and observed market shares derived from absolute error (Cherchi and Cirillo 2010) was used in this test. It can be expressed as:

$$
2-\text { norms }=\sqrt{D_{A l t .1}^{2}+\cdots D_{A l t . i}^{2}}
$$

where $D_{\text {Alt.i }}$ means the absolute error between observed and predicted shares for alternative $i$. Note that if the predicted and observed market shares are the same, D is equal to zero. It becomes bigger if the difference between predicted and observed market share increases. Thus, by definition, a lower value of the 2-norms statistic indicates a higher predictive power.

\section{Case study 1: Stated choice experiment}

\section{Data}

The first case study is based on a stated choice experiment constructed to analyze consumer choice of shopping center. The experiment described shopping centers in terms of three generic attributes - travel time, parking fees, and number of stores. Each attribute was categorized into four levels. Travel time was labeled 10, 30, 50 and 70 min, parking costs were varied in terms of $0.5,1.0,1.5,2.0$ euros, while levels for the number of stores were 30, 60, 90, and 120 stores respectively. Three imaginary shopping centers were considered as alternatives in choice sets. A $4^{9}$ orthogonal fractional factorial choice set design consisting of 128 runs was constructed to orthogonally vary the attributes within and between the shopping centers. These choice set were blocked into 16 choice sets. A total of 242 participants indicated for each of these choice sets which of these three centers they would choose. ${ }^{2}$

\section{Estimation results}

\section{Original model}

The original regret model (Eq. 3) was compared with the logarithmic specification (Eqs. 6) to figure out how well each base regret model represents observed consumer choice

\footnotetext{
1 The test provides upper bound using the standard normal cumulative distribution of probabililty that a non-nested model superior to another.

2 The choice card is available on request from the authors.
} 
Table 2 Summary of estimation results-original models: case study 1

\begin{tabular}{lll}
\hline Shopping location choice & Original regret & Logarithmic specification \\
\hline Travel time & -0.0592 & -0.0371 \\
$(t$ value $)$ & $(-36.45)$ & $(-34.31)$ \\
Parking fee & -1.3415 & -0.8103 \\
$(t$ value $)$ & $(-25.67)$ & $(-22.99)$ \\
Number of stores & 0.0099 & 0.0073 \\
$(t$ value $)$ & $(12.23)$ & $(14.18)$ \\
$\mathrm{R}^{2}$ & 0.327 & 0.317 \\
Adj. $\mathrm{R}^{2}$ & 0.326 & 0.316 \\
Final log-likelihood & -2863.021 & -2907.148 \\
\hline
\end{tabular}

behavior. Recall that the regret functions are identical for larger attribute difference, but differ for smaller differences. Moreover, whereas the original regret model is based on a comparison of the best foregone only choice alternative, the logarithmic specification, considered in this study is based on all pairwise comparisons. Thus, the analysis is a partial replication of an earlier study, using a different data set (Rasouli and Timmermans 2015a). Although this comparison is not the focus on attention in this study, we included the results because they add empirical knowledge to question which specification seems behaviorally the best. The findings of the estimation results of each model are listed in Table 2.

All coefficients are statistically significant at the 95 percent significance level and their sign is in anticipated direction. The absolute values of the attribute parameters are larger in the original regret model than in logarithmic specification, as expected. Parking fees is the most influential attribute in both models. This result may be affected by the attribute range since parking fees has the smallest attribute range, compared to other attributes. The original formulation outperforms Chorus' later specification, consistent with the result of previous research (Rasouli and Timmermans 2015a). The results of Ben-Akiva and Swait's test (1986) for non-nested choice models indicate that the probability that the logarithmic specification outperforms the original regret is zero $(p \leq \Phi(-9.224) \cong 0)$. $(\Phi)$ is the standard normal cumulative distribution.

\section{Specification based on Weber's law}

In this section, we discuss the results of the comparison of the model specifications that include Weber's law with their original, base specifications. Table 3 shows the estimation results and the difference in predictive performance of the models.

The $t$ values indicate the parameters are statistically significant at the $95 \%$ significance level. Their signs are consistent with theoretical anticipations. By applying Weber's law, the absolute values of the parameters are increased in both regret models. This seems reasonable since the attribute difference is divided by the attribute value, and therefore larger parameters are needed to represent similar attribute level regret. The increment is smaller for parking fees than for other attributes, which may reflect the effect of attribute range. That is, since the original regret model does not consider the attribute range, and parking fees has the smallest range, the absolute value of the parameter for parking fees is relatively high. However, by applying Weber's law, attribute values in the denominator become normalized. As a result, while parking fees is the most influential attribute in the original regret model, travel time becomes most important when applying Weber's law. 
Table 3 Summary of estimation results-specification based on Weber's law: case study 1

\begin{tabular}{|c|c|c|c|c|}
\hline \multirow[t]{2}{*}{ Shopping location choice } & \multicolumn{2}{|c|}{ Original regret } & \multicolumn{2}{|c|}{ Logarithmic specification } \\
\hline & $\begin{array}{l}\text { Base } \\
\text { formulation }\end{array}$ & $\begin{array}{l}\text { Specification based on } \\
\text { Weber's law }\end{array}$ & $\begin{array}{l}\text { Base } \\
\text { formulation }\end{array}$ & $\begin{array}{l}\text { Specification based on } \\
\text { Weber's law }\end{array}$ \\
\hline $\begin{array}{l}\text { Travel time } \\
(t \text { value })\end{array}$ & $\begin{array}{l}-0.0592 \\
(-36.45)\end{array}$ & $\begin{array}{l}-3.1425 \\
(-37.31)\end{array}$ & $\begin{array}{l}-0.0371 \\
(-34.31)\end{array}$ & $\begin{array}{l}-1.3742 \\
(-26.06)\end{array}$ \\
\hline $\begin{array}{l}\text { Parking fee } \\
(t \text { value })\end{array}$ & $\begin{array}{l}-1.3415 \\
(-25.67)\end{array}$ & $\begin{array}{l}-2.3502 \\
(-26.21)\end{array}$ & $\begin{array}{l}-0.8103 \\
(-22.99)\end{array}$ & $\begin{array}{l}-1.3691 \\
(-26.06)\end{array}$ \\
\hline $\begin{array}{l}\text { Number of stores } \\
(t \text { value })\end{array}$ & $\begin{array}{l}0.0099 \\
(12.23)\end{array}$ & $\begin{array}{l}0.3448 \\
(12.98)\end{array}$ & $\begin{array}{l}0.0073 \\
(14.18)\end{array}$ & $\begin{array}{l}0.3284 \\
(15.81)\end{array}$ \\
\hline $\mathrm{R}^{2}$ & 0.327 & 0.330 & 0.317 & 0.319 \\
\hline Adj. $R^{2}$ & 0.326 & 0.329 & 0.316 & 0.319 \\
\hline Final log-likelihood & -2863.021 & -2849.527 & -2907.148 & -2895.728 \\
\hline
\end{tabular}

Based on the results of Ben-Akiva and Swait's test (1986) for non-nested choice models, we can conclude that the regret model, which includes the non-proportional perception effect, performs better than the original formulations (original regret and logarithmic specification). The probability that the original regret model is superior to its specification based on the Weber's law is zero $(p \leq \Phi(-5.052) \cong 0$, with $\Phi$ being the standard normal cumulative distribution function). Also, the probability that the logarithmic specification is superior to its specification based on Weber's law is zero $(p \leq \Phi(-4.125) \cong 0)$.

Compared to the base formulations of the regret models, the specifications based on Weber's law slightly improve the goodness-of-fit of the models. Thus, it seems that in this case study, the inclusion of the mapping of objectives values into cognitive attributes results in better predictive performance of the regret models.

\section{Specification based on generalized Weber's law}

Focusing on the specification based on the generalized Weber's law, similar results were obtained for both regret models (original regret and logarithmic specification). Table 4 shows the estimation results. The power coefficient for the number of stores in the modified Weber model specification is not statistically significant at the 95 percent significance level in both regret models. Therefore, they were dropped and replaced by one as in Weber's Law.

Applying the generalized Weber's law, the perception effect is somewhat diminished. It turns out that the increment in the absolute values of the estimated parameters is slightly lower than the specification based on Weber's law. Parking fees is the most influential attribute in both regret models based on the generalized Weber's law, while travel time is the most influential attribute in the specification based on Weber's law.

The value of the power coefficient in the specification based on the generalized Weber's law is about 0.4 in the case of the original regret model, which is slightly smaller than in the case of the logarithmic specification (the value of the power coefficient is around 0.5). These results are congruent with Guilford's argument (1932) that the power coefficient should have a value between zero and one.

All specifications incorporating the generalized Weber's law outperform their base model specifications according to Ben-Akiva and Swait (1986)'s test for non-nested choice 
Table 4 Summary of estimation results-specification based on Weber's law: case study 1

\begin{tabular}{|c|c|c|c|c|}
\hline \multirow{2}{*}{$\begin{array}{l}\text { Shopping location } \\
\text { choice }\end{array}$} & \multicolumn{2}{|c|}{ Original regret } & \multicolumn{2}{|c|}{ Logarithmic specification } \\
\hline & $\begin{array}{l}\text { Base } \\
\text { formulation }\end{array}$ & $\begin{array}{l}\text { Specification based on } \\
\text { generalized Weber's law }\end{array}$ & $\begin{array}{l}\text { Base } \\
\text { formulation }\end{array}$ & $\begin{array}{l}\text { Specification based on } \\
\text { generalized Weber's law }\end{array}$ \\
\hline $\begin{array}{l}\text { Travel time } \\
(t \text { value })\end{array}$ & $\begin{array}{l}-0.0592 \\
(-36.45)\end{array}$ & $\begin{array}{l}-0.2788 \\
(-3.44)\end{array}$ & $\begin{array}{l}-0.0371 \\
(-34.31)\end{array}$ & $\begin{array}{l}-0.2313 \\
(-3.19)\end{array}$ \\
\hline $\begin{array}{l}\text { Parking fee } \\
(t \text { value })\end{array}$ & $\begin{array}{l}-1.3415 \\
(-25.67)\end{array}$ & $\begin{array}{l}-1.7152 \\
(-11.99)\end{array}$ & $\begin{array}{l}-0.8103 \\
(-22.99)\end{array}$ & $\begin{array}{l}-1.1043 \\
(-19.29)\end{array}$ \\
\hline $\begin{array}{l}\text { Number of stores } \\
\text { ( } t \text { value })\end{array}$ & $\begin{array}{l}0.0099 \\
(12.23)\end{array}$ & $\begin{array}{l}0.3333 \\
(12.42)\end{array}$ & $\begin{array}{l}0.0073 \\
(14.18)\end{array}$ & $\begin{array}{l}0.3215 \\
(14.98)\end{array}$ \\
\hline $\begin{array}{l}\text { Power } \\
\text { Travel time } \\
(t \text { value })\end{array}$ & & $\begin{array}{l}0.3811 \\
(5.30)\end{array}$ & & $\begin{array}{l}0.4714 \\
(5.82)\end{array}$ \\
\hline $\begin{array}{l}\text { Power } \\
\text { Parking fee } \\
(t \text { value })\end{array}$ & & $\begin{array}{l}0.4083 \\
(3.02)\end{array}$ & & $\begin{array}{l}0.5648 \\
(4.74)\end{array}$ \\
\hline $\mathrm{R}^{2}$ & 0.327 & 0.339 & 0.317 & 0.327 \\
\hline Adj. $\mathrm{R}^{2}$ & 0.326 & 0.338 & 0.316 & 0.326 \\
\hline Final log-likelihood & -2863.021 & -2813.136 & -2907.148 & -2862.776 \\
\hline
\end{tabular}

models. The probability that the original regret model outperforms its elaboration based on the generalized Weber's law is zero $(p \leq \Phi(-10.005) \cong 0)$, and the probability that the logarithmic specification is superior to its elaboration based on the generalized Weber's law is also zero $(p \leq \Phi(-9.115) \cong 0)$. The improvement in goodness-of-fit of the models based on the generalized Weber's law is higher than the improvement based on the classic Weber's law.

\section{Validation results}

A K-fold cross-validation test was conducted to validate the estimation results. In total, 11 subsets $(\mathrm{K}=11)$ were constructed, randomly assigning the respondents to the subsets. Of the 11 subsets, 10 subsets were used to estimate the models, while the one remaining subset was used to validate the estimation results. This process is repeated 11 times using all subsets as validation data.

\section{Original models}

First, the cross-validation test is applied to original regret model and the logarithmic specification. Results show that the estimated parameters only slightly differ between the 11 subsets. Likewise, the goodness-of-fit of the models differs only marginally. This implies that the data are very stable. The validation step involves using the estimated model to predict the observations in the 11 th subset.

Figure 1 shows the 2-norms values of both regret models, which quantify the predictive success of the estimated model. Akin to the estimation results for the full data set, the results of the 11 fold cross-validation test indicate that the original regret model is superior to the logarithmic specification. A few fluctuations can be observed. Thus, we can rule out that a peculiar subset or peculiar respondent causes the improvement in predictive success of the original regret model. 


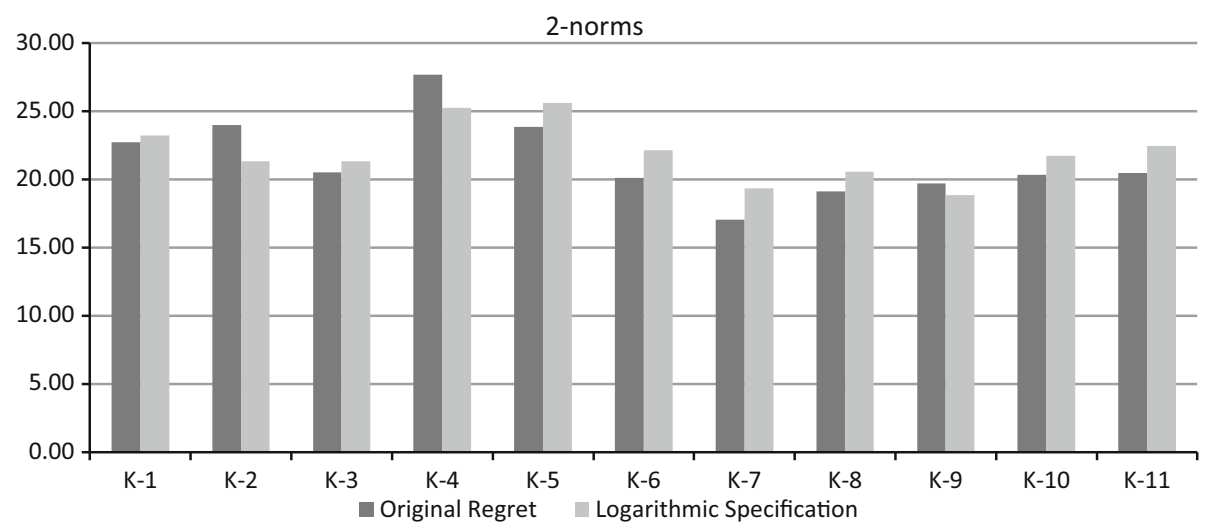

Fig. 1 2-norms values for K-fold cross-validation test: Comparison of original regret and logarithmic specification—case study 1

\section{Specification based on Weber's law}

Second, each regret model (original regret and logarithmic specification) and their variants based on Weber's law were subjected to the cross-validation test. The estimated parameters and their goodness-of-fit only show small variations between the 11 training data sets. This implies that this data set is suitable to conduct the cross-validation test.

The 2-norms values for the 11-fold cross-validation are shown in Fig. 2. It demonstrates that the variant based on Weber's law performs better in predicting observed choice behavior. This means that when regret is defined to include the psycho-physical relationship, the model is superior to its original base specification. Compared to the base formulations, the value of 2-norms is generally lower for the models specifications based on weber's law, because the model fit is better.

\section{Specification based on the generalized Weber's law}

Finally, the 11-fold cross-validation test was applied to the specification based on the generalized Weber's law. The parameters are similar and the goodness-of-fit of the models is almost constant across training sets. Figure 3 presents the values of 2-norms. The variants based on the generalized Weber's law show lower values than their original formulations, implying a higher predictive ability. The difference in predictive ability of the models is much clearer than in case of Weber's law. The 2-norms values of the variants based on the generalized Weber's law show lower values than the original formulations.

\section{Case study 2: revealed choice experiment}

\section{Data}

For the second case study, we used the 2009 MON data (Mobiliteit Onderzoek Nederlands - the Dutch National Travel Survey). The survey was administered among representative residents in the Netherlands to obtain daily travel information. From the complete revealed preference (RP) data set, we extracted the data of the Noord Brabant Province, 


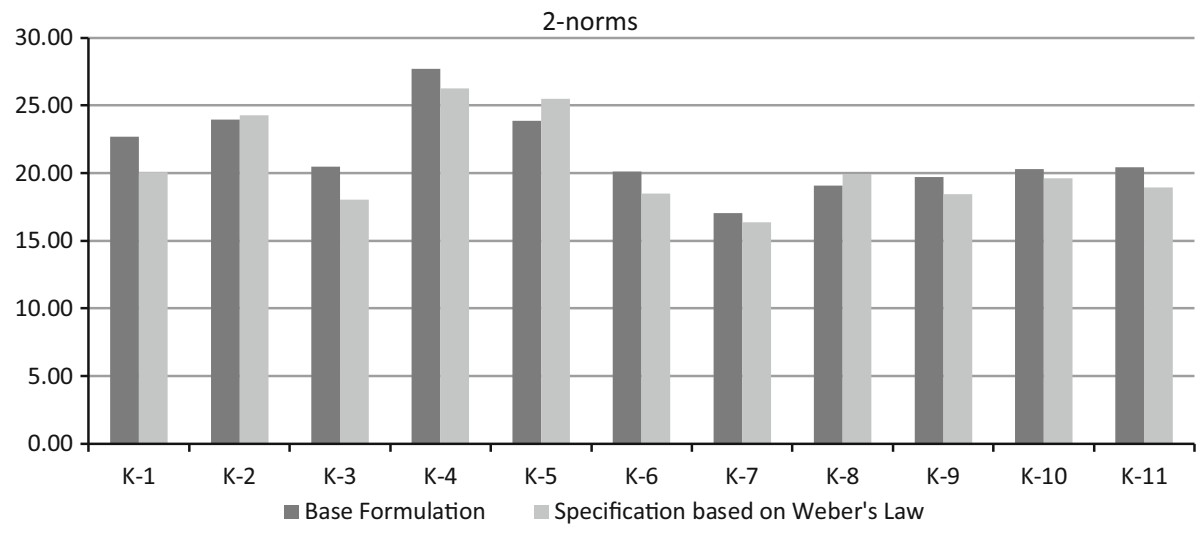

(a)

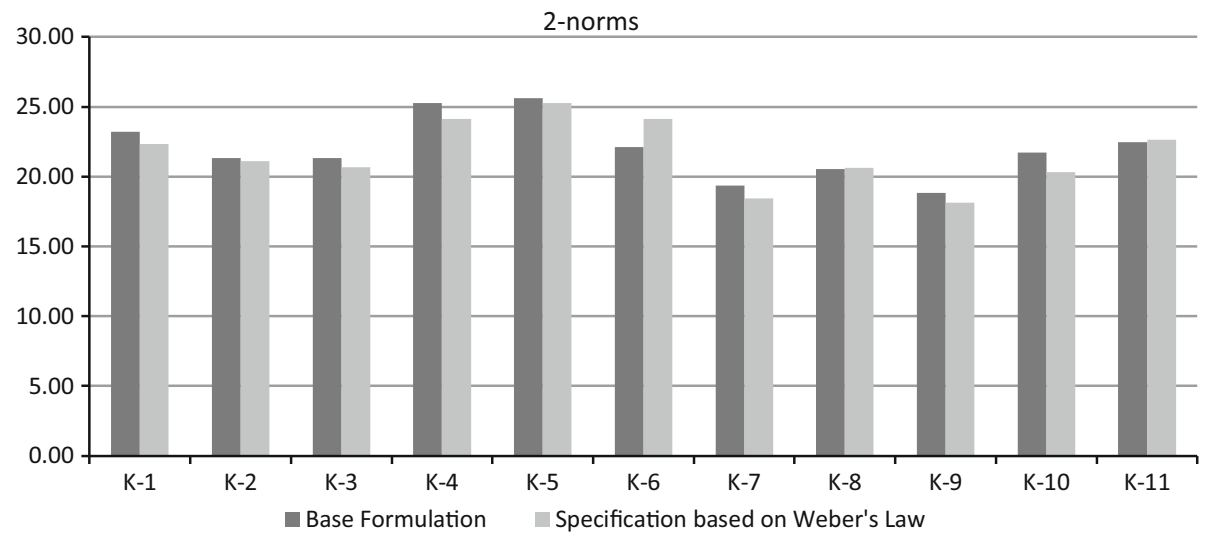

(b)

Fig. 2 2-norms values for K-fold cross-validation test: Comparison of base formulations and their variants based on Weber's law-Case study 1. a Original regret and its variant based on Weber's law. b Logarithmic specification and its variant based on Weber's law

which concerns 354 respondents. The data includes two level of service variables (travel time and travel distance) for three mode choice alternatives (car, bike, walk). The travel time ranges from 1 to $134 \mathrm{~min}$, while travel distance varies from 1 to $91 \mathrm{~km}$. The variance of travel time is 56.48, which is larger than the variance of travel distance (50.89). The estimated models thus predict the probability of mode choice as a function of these level of service variables.

\section{Estimation results}

As in the first case study, the original regret model, the logarithmic regret model and their variants based on the Weber's law and the generalized Weber's law were estimated. The primary aim of the analysis was to examine and statistically test whether model specifications that including a mapping of the objective attribute values into cognitive values outperform their original specifications. Implicitly, this also allows examining whether the 


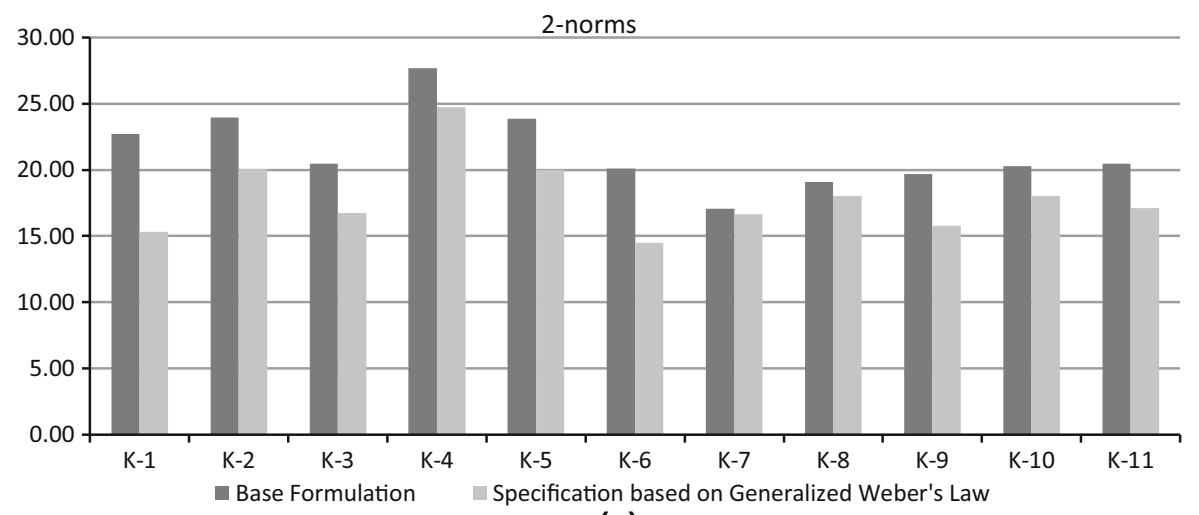

(a)

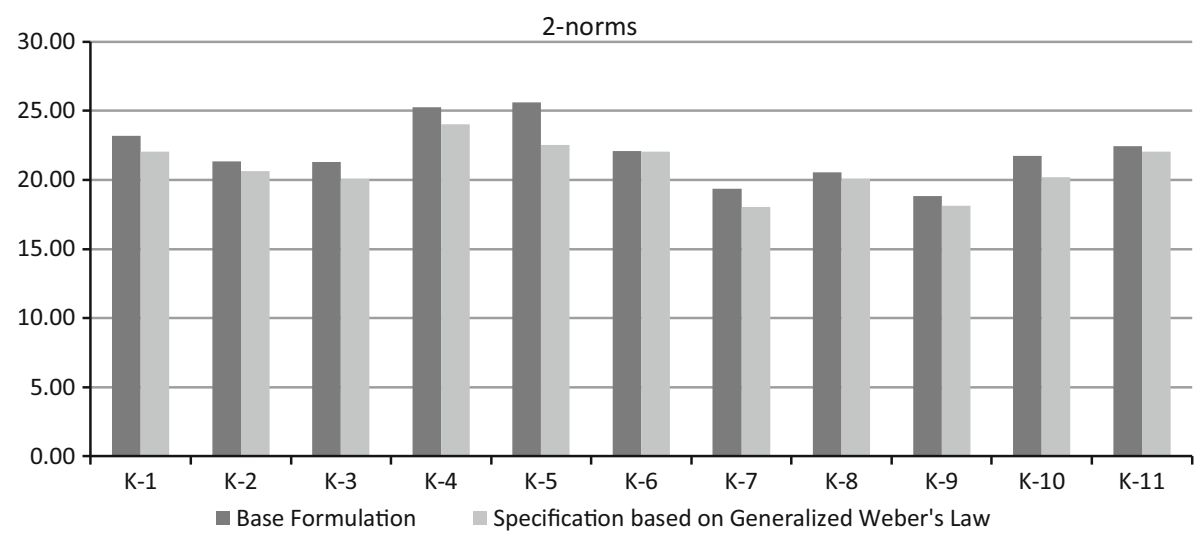

(b)

Fig. 3 2-norms values for K-fold cross-validation test: comparison of base formulations and their variants based on the generalized Weber's law-case study 1. a Original regret and its variant based on the generalized Weber's law. b Logarithmic specification and its variant based on the generalized Weber's law

mode based on the generalized Weber specification outperform the model, based on the Weber's law, and whether the logarithmic model outperforms the original regret model, introduced in the transportation research community by Chorus et al. (2008a, b).

\section{Original models}

Table 5 shows the detailed estimation results for the original regret and the logarithmic regret models. It shows that the overall results are similar to those obtained in the first case study. All estimated coefficients are statistically significant at the $95 \%$ significance level and the signs are in anticipated direction. The logarithmic specification has smaller parameters than the original regret model; the same as in the first case study. Travel distance has more influence on choice than travel time in both regret models. Results also clearly indicate again that the original regret model is superior to the logarithmic specification in terms of predictive ability. Based on the non-nested choice model test (Ben- 
Table 5 Summary of estimation results: base formulations-case study 2

\begin{tabular}{lll}
\hline Mode choice & Original regret & Logarithmic specification \\
\hline Travel time & -0.1152 & -0.0711 \\
$(t$ value $)$ & $(-14.97)$ & $(-14.02)$ \\
Travel distance & -0.5222 & -0.3563 \\
$(t$ value $)$ & $(-13.01)$ & $(-12.81)$ \\
$\mathrm{R}^{2}$ & 0.202 & 0.187 \\
Adj. $\mathrm{R}^{2}$ & 0.200 & 0.185 \\
Final log-likelihood & -1015.838 & -1034.352 \\
\hline
\end{tabular}

Akiva and Swait 1986), the probability that the logarithmic specification outperforms the original regret model is zero $(p \leq \Phi(-6.178) \cong 0)$.

\section{Specification based on Weber's law}

Next, we compare the performance of the two model specifications that include the Weber's law against their original model specifications. The results are shown in Table 6. As in the first case study, the predictive performance of the models indicates that the models incorporating the psycho-physical relationship outperform the base models, but the degree of improvement is higher than in the first case study. This may be due to the fact that in the second case study, the attributes of all alternatives have to be profoundly perceived rather than explicitly be shown to the respondents as in the first case study. Therefore, the difference between cognitive representation of attribute values and their objective counterparts are likely to be larger, reflected by the higher improved performance of the model incorporating this phenomena.

All parameters are statistically significant at the $95 \%$ significance level, and the signs of all parameters are in anticipated direction. The absolute value of the parameters is increased compared to the base models. Travel distance is more influential than travel time in all models. The results of the non-nested choice model test (Ben-Akiva and Swait 1986) reveal that the specifications based on Weber's law better predict observed choice behavior than the base formulations. The probability that the original regret model is superior to its specification based on the Weber's law is zero $(p \leq \Phi(-4.785) \cong 0)$, and the probability that the logarithmic specification outperforms its specification based on Weber's law is also zero $(p \leq \Phi(-3.567) \cong 0)$.

Table 6 Summary of estimation results: specification based on Weber's law-case study 2

\begin{tabular}{|c|c|c|c|c|}
\hline \multirow{2}{*}{ Mode choice } & \multicolumn{2}{|l|}{ Original regret } & \multicolumn{2}{|c|}{ Logarithmic specification } \\
\hline & Base formulation & $\begin{array}{l}\text { Specification based } \\
\text { on Weber's law }\end{array}$ & $\begin{array}{l}\text { Base } \\
\text { formulation }\end{array}$ & $\begin{array}{l}\text { Specification based } \\
\text { on Weber's law }\end{array}$ \\
\hline $\begin{array}{l}\text { Travel time } \\
(t \text { value })\end{array}$ & $\begin{array}{l}-0.1152 \\
(-14.97)\end{array}$ & $\begin{array}{l}-2.4107 \\
(-16.08)\end{array}$ & $\begin{array}{l}-0.0711 \\
(-14.02)\end{array}$ & $\begin{array}{l}-1.3731 \\
(-17.36)\end{array}$ \\
\hline $\begin{array}{l}\text { Travel distance } \\
(t \text { value })\end{array}$ & $\begin{array}{l}-0.5222 \\
(-13.01)\end{array}$ & $\begin{array}{l}-7.0834 \\
(-14.45)\end{array}$ & $\begin{array}{l}-0.3563 \\
(-12.81)\end{array}$ & $\begin{array}{l}-5.2538 \\
(-12.27)\end{array}$ \\
\hline $\mathrm{R}^{2}$ & 0.202 & 0.211 & 0.187 & 0.192 \\
\hline Adj. $R^{2}$ & 0.200 & 0.210 & 0.185 & 0.190 \\
\hline Final log-likelihood & -1015.838 & -1003.336 & -1034.352 & -1030.254 \\
\hline
\end{tabular}


Table 7 Summary of estimation results: specification based on the generalized Weber's law-case study 2

\begin{tabular}{|c|c|c|c|c|}
\hline \multirow[t]{2}{*}{ Mode choice } & \multicolumn{2}{|c|}{ Original regret } & \multicolumn{2}{|c|}{ Logarithmic specification } \\
\hline & $\begin{array}{l}\text { Base } \\
\text { formulation }\end{array}$ & $\begin{array}{l}\text { Specification based on the } \\
\text { generalized Weber's law }\end{array}$ & $\begin{array}{l}\text { Base } \\
\text { formulation }\end{array}$ & $\begin{array}{l}\text { Specification based on } \\
\text { the generalized } \\
\text { Weber's law }\end{array}$ \\
\hline $\begin{array}{l}\text { Travel time } \\
(t \text { value })\end{array}$ & $\begin{array}{l}-0.1152 \\
(-14.97)\end{array}$ & $\begin{array}{l}-0.5237 \\
(-13.41)\end{array}$ & $\begin{array}{l}-0.0711 \\
(-14.02)\end{array}$ & $\begin{array}{l}-0.1987 \\
(-3.81)\end{array}$ \\
\hline $\begin{array}{l}\text { Travel distance } \\
(t \text { value })\end{array}$ & $\begin{array}{l}-0.5222 \\
(-13.01)\end{array}$ & $\begin{array}{l}-4.0936 \\
(-11.97)\end{array}$ & $\begin{array}{l}-0.3563 \\
(-12.81)\end{array}$ & $\begin{array}{l}-3.4025 \\
(-4.21)\end{array}$ \\
\hline $\begin{array}{l}\text { Power_Travel time } \\
(t \text { value })\end{array}$ & & $\begin{array}{l}0.7381 \\
(10.35)\end{array}$ & & $\begin{array}{l}0.7903 \\
(5.12)\end{array}$ \\
\hline $\begin{array}{l}\text { Power_Travel distance } \\
(t \text { value })\end{array}$ & & $\begin{array}{l}0.7169 \\
(8.72)\end{array}$ & & $\begin{array}{l}0.7752 \\
(7.49)\end{array}$ \\
\hline $\mathrm{R}^{2}$ & 0.202 & 0.225 & 0.187 & 0.203 \\
\hline Adj. $\mathrm{R}^{2}$ & 0.200 & 0.222 & 0.185 & 0.202 \\
\hline Final log-likelihood & -1015.838 & -986.297 & -1034.352 & -1013.836 \\
\hline
\end{tabular}

\section{Specification based on the generalized Weber's law}

When the regret models (original regret and logarithmic specification) are specified based on the generalized Weber's law, their predictive performance is improved. Again, this finding is consistent with the results obtained in the first case study. Table 7 shows the results. Similar to the specification based on Weber's law, the improvement is higher in the second case study. The probability that the original regret model outperforms the specification based on the generalized Weber's law is zero $(p \leq \Phi(-7.518) \cong 0)$. Similarly the probability that the logarithmic specification is superior to the variant based on the generalized Weber's law is zero $(p \leq \Phi(-6.222) \cong 0)$.

The power coefficient for travel time and distance is somewhat higher in the logarithmic specification. It is slightly over 0.7 for the original regret model, and close to 0.8 for the logarithmic specification. These values are closer to 1 than in the first case study meaning that the difference between objective attribute difference and cognitive representation is larger in the second case study incurred by the type of choice mechanism. (between revealed preference and stated choice experiment).

\section{Validation results}

The models estimated in this second case study were also validated using the K-fold crossvalidation method. To that end, the data were randomly split into 6 different subsets. The models were estimated pooling 5 of these subsets, and the estimated model was used to predict the observations in the 6th subset. By repeating this procedure 6 times, each subset is used for validating the model once.

\section{Original models}

First, the original regret and the logarithmic specification were put to the cross-validation test. Estimation results across the 5 subsets are similar to the estimation results of the full data set. The values of the parameters are close and the original specification of the regret 


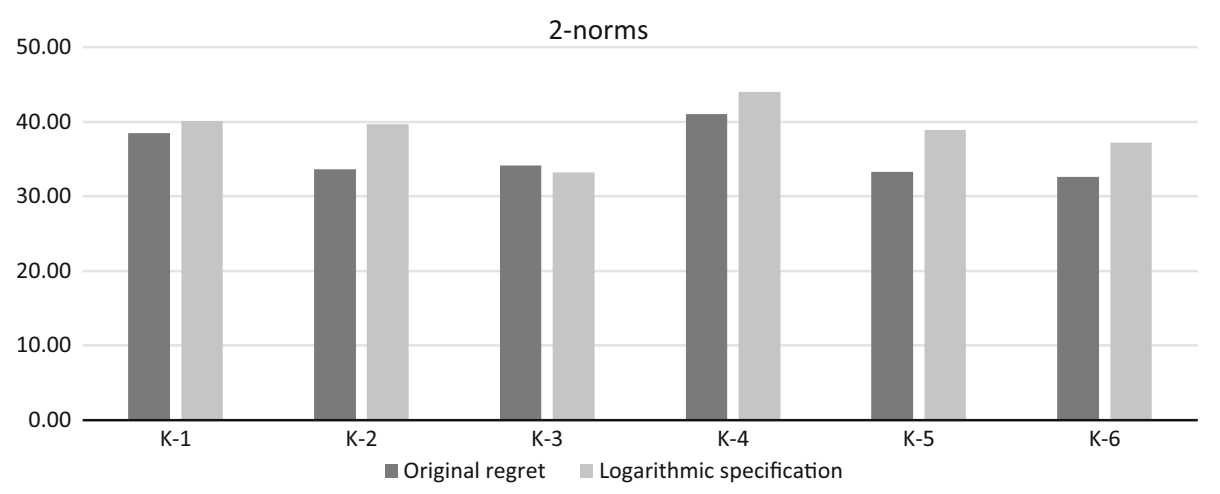

Fig. 4 2-norms values for K-fold cross-validation test: Comparison of original regret and logarithmic specification-case study 2

model consistently outperforms the later introduced logarithmic specification. The validation results are presented in Fig. 4. It demonstrates that the original regret model is superior to the logarithmic specification.

\section{Specification based on Weber's law}

Second, the cross validation test was applied to the base formulations of each regret model and their variants based on Weber's law. The estimated models are similar across the training sets. Figure 5 shows the validation result in terms of the 2 -norms. The overall results are similar to those obtained for the first case study, but the dominance relationship between the models is clearer. Except for small fluctuations, the specifications based on Weber's law are superior to the corresponding base formulations of the two regret models.

\section{Specification based on the generalized Weber's law}

Lastly, each regret model and their perception-based variants based on the generalized Weber's law were subjected to the cross-validation test. The full data set seems suitable to estimate the parameters and assess model fit. The validation results using the 2-norms statistic are shown in Fig. 6. Each variant is found to have a lower value, meaning that their predictive power is better than that of the base formulations. The results reinforce the findings of the first case study. Incorporating the psycho-physical relationship into the regret model improves their predictive ability. The difference between the values of 2-norms is larger than for the specification based on Weber's law.

\section{Conclusions and discussion}

This paper has documented the formulation and empirical test of new regret-based choice models. The new models differs from their original, base formulations that mark the evolution of regret-based models in transportation research in that regret is not defined in terms of attributes differences between the considered choice alternative and the (best) non-chosen choice alternatives, but rather as the ratio of attribute differences to the 


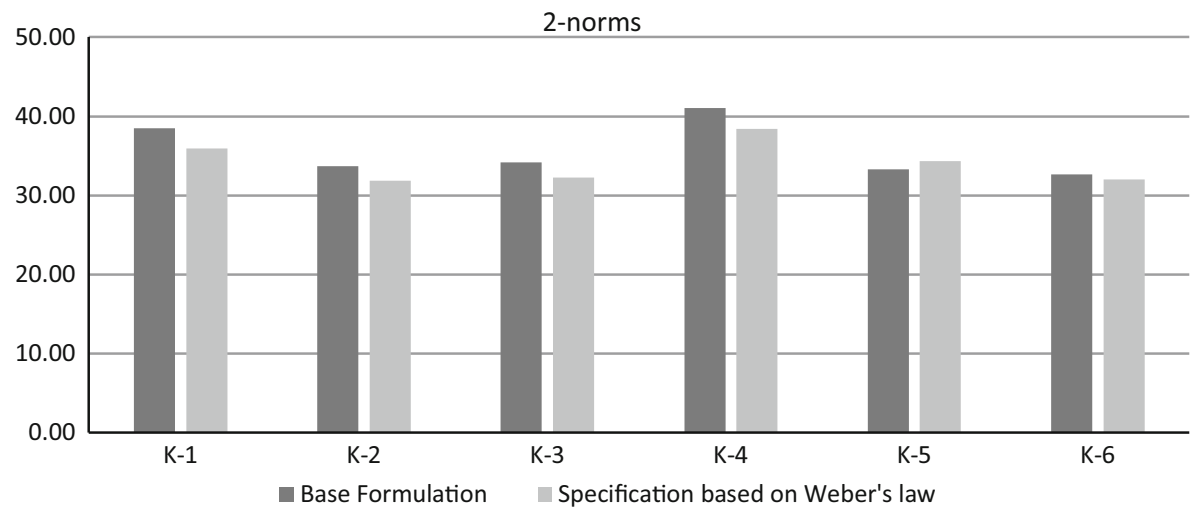

(a)

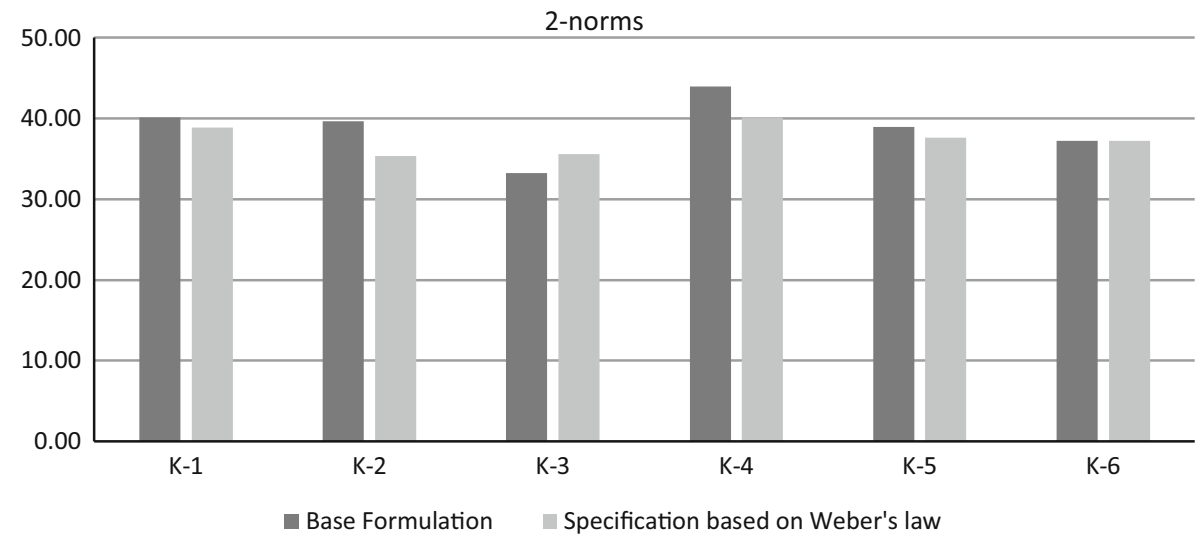

(b)

Fig. 5 2-norms values for K-fold cross-validation test: comparison of original formulations and their variant based on Weber's law-case study 2. a Original regret and its variant based on Weber's law. b Logarithmic specification and its variant based on Weber's law

absolute attribute values of the considered alternative. This transformation is assumed to represent the mapping of objective attribute values into cognitive attributes. Two specifications were tested: model variants incorporating the classic Weber's law and model variants based on the generalized Weber's law. These specifications are motivated by the results of psycho-physical research which has shown that the perception of change in a stimuli is relative to the absolute intensity of the stimuli. Although this research has traditionally focused on physical stimuli such as noise and sound, the perception of other stimuli may be driven by similar perceptual mechanisms.

The results of two case studies, one based on stated choice data, the other on revealed choice data, convincingly and consistently show that for both the original best only regret model and the paired comparison logarithmic specification of the regret model, incorporating the mapping function significantly outperform the original base specifications. These findings thus support the conclusion that it is crucial to account for the size of attributes of alternatives in addition to the attribute difference in regret-based models (and maybe in utility-based models as well) to better capture choice behavior. The evidence from the two 


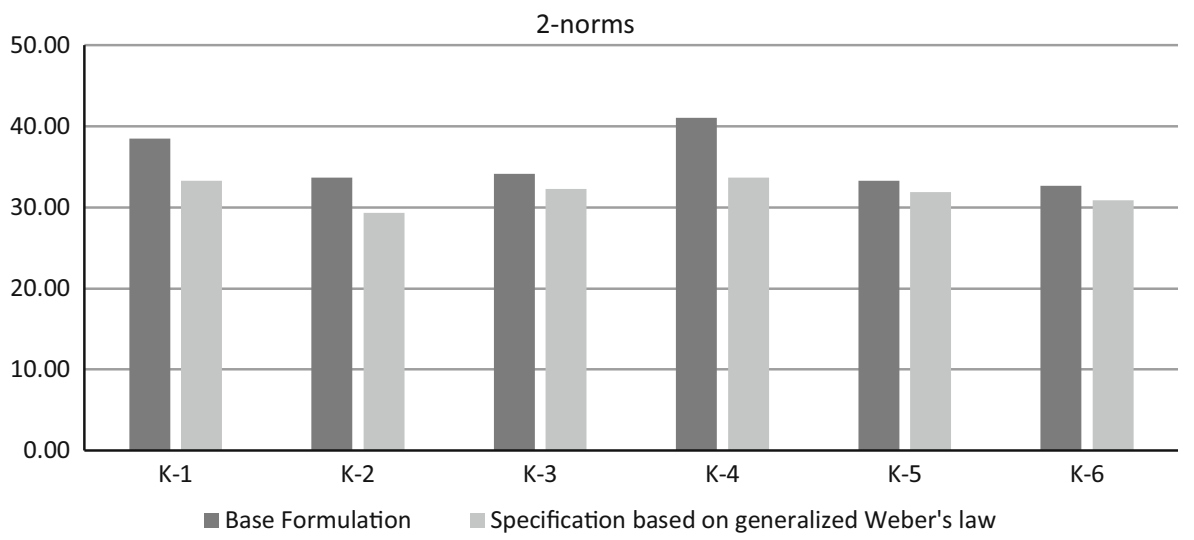

(a)

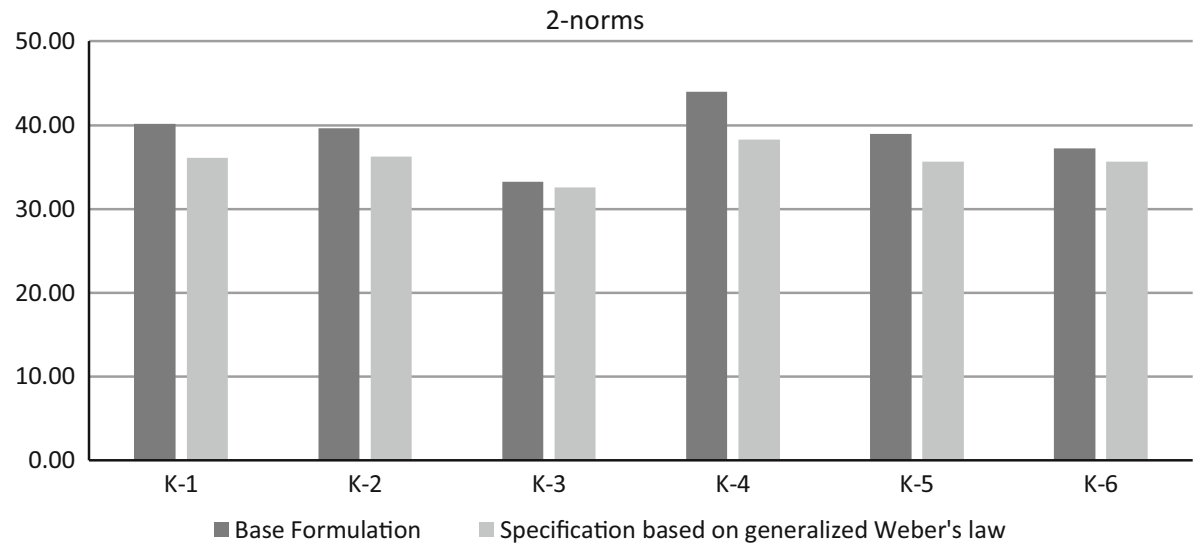

(b)

Fig. 6 2-norms values for the K-fold cross-validation test: comparison of original formulations and their variants based on the generalized Weber's law-case study 2. a Original regret and its variant based on the generalized Weber's law. b Logarithmic specification and its variant based on the generalized Weber's law

case studies clearly shows that the inclusion of the perception component in the specification of the regret function outperforms the original random regret models. Further evidence is provided by the results of the K-fold cross-validation, which shows that the overall results also hold for the various subsets.

Moreover, results of the model comparisons demonstrate that the most general model, the one based on the generalized Weber's law, performs best. This is not surprising in the sense that the classic Weber is a special case of the generalized Weber's law. However, results indicate that the models based on the generalized Weber's law perform significantly better and that the extra estimated parameter in the generalized function is not equal to one.

Finally, as a by-product of this study, these two case studies replicate the findings of another study (Rasouli and Timmermans 2015a), which concluded that the original regret model, based on the max operator outperforms the later introduced logarithmic specification. Of course, further empirical analyses are needed to increase our body of knowledge regarding the empirical performance of different regret models. 
The main contribution of this paper is the attempt to include a perceptual mechanism in the formulation of regret models. It is argued that if perception involves a non-linear mapping of physical attribute values into cognitive values, commonly used regret-minimization models lead to less accurate results, which can be mitigated by considering the size of attributes of each alternative in the regret formulation. It goes without saying that this conclusion is based on these two case studies only. It would be interesting and valuable to replicate the comparison on other data to judge the generalizability of the current findings to different data sets and spatial and cultural settings. For the case of stated choice data, it would be interesting to see to what extent results depend on the size of attribute variation.

The results reported in this paper have been deliberately limited to the two pure random regret models. The evolution of regret-based models in transportation research however also includes attempt to formulate mixed utility/regret models Hess et al. (2012), Hess and Stathopoulos (2013) and Boeri et al. (2014). Further research should analyze whether the inclusion of the psycho-physical relationship will also improve the predictive success of other regret-based or hybrid models (e.g. Chorus 2014b).

Open Access This article is distributed under the terms of the Creative Commons Attribution 4.0 International License (http://creativecommons.org/licenses/by/4.0/), which permits unrestricted use, distribution, and reproduction in any medium, provided you give appropriate credit to the original author(s) and the source, provide a link to the Creative Commons license, and indicate if changes were made.

\section{References}

Beck, M.B., Chorus, C.G., Rose, J.M., Hensher, D.A.: Vehicle purchasing behaviour of individuals and groups. Transp. Econ. Policy. 47(47), 475-492 (2013)

Bell, D.E.: Regret in decison making under uncertainty. Oper. Res. 30(2), 961-981 (1982)

Ben-Akiva, M., Swmt, J.: The Akaike likelihood ratio index. Transp. Sci. 20, 133-136 (1986)

Boeri, M., Masiero, L.: Regret Minimization and utility maximization in a freight transport context: an application from two stated choice experiments. Transp. A 10(6), 548-560 (2014)

Boeri, M., Scarpa, R., Chorus, C.G.: Stated choices and benefit estimates in the context of traffic calming schemes: utility maximization, regret minimization, or both? Transp. Res. Part A Policy Pract. 61, 121-135 (2014)

Cherchi, E., Cirillo, C.: Validation and forecasts in models estimated from multiday travel survey. Transp. Res. Rec. 2175, 57-64 (2010)

Chorus, C.G.: A new model of random regret minimization. Ejtir 10(2), 181-196 (2010)

Chorus, C.G.: Random regret minimization: an overview of model properties and empirical evidence. Transp. Rev. 32(1), 75-92 (2012a)

Chorus, C.G.: Logsums for utility-maximizers and regret-minimizers, and their relation with desirability and satisfaction. Transp. Res. Part A Policy Pract. 46, 1003-1012 (2012b)

Chorus, C.G., Bierlaire, M.: An empirical comparison of travel choice models that capture preferences for compromise alternatives. Transportation 40, 549-562 (2013)

Chorus, C.G.: Benefit of adding an alternative to one's choice set: a regret minimization perspective. J. Choice Model. 13, 1-11 (2014a)

Chorus, C.G.: Acquisition of ex-post travel information: a matter of balancing regrets. Transp. Sci. 48(2), 243-255 (2014b)

Chorus, C.G.: A generalized random regret minimization model. Transp. Res. Part B 68, 224-238 (2014c)

Chorus, C.G., Arentze, T.A., Timmermans, H.J.P.: A random regret-minimization model of travel choice. Transp. Res. Part B 42, 1-18 (2008a)

Chorus, C.G., Arentze, T.A., Timmermans, H.J.P.: A Comparison of Rgret-minimization and utility-maximization in the context of travel mode-choices. In: Proceedings of 87 th annual meeting of the Transportation Research Board, Washington, DC (2008b)

Chorus, C.G., de Jong, G.C.: Modeling experienced accessibility for utility-maximizers and regret-minimizers. J. Transp. Geogr. 19, 1155-1162 (2011) 
Chorus, C.G., Rose, J.M., Hensher, D.A.: Regret minimization or utility maximization: it depends on the attribute. Environ. Plan. B Plan. Des. 40, 154-169 (2013)

De Bekker-Grob, E.W., Chorus, C.G.: Random regret-based discrete-choice modelling: an application to healthcare. Pharmacoeconomics 31(7), 623-634 (2013)

De Moraes Ramos, G., Daamen, W., Hoogendoorn, S.: Expected utility theory, prospect theory, and regret theory compared for prediction of route choice behavior. Transp. Res. Rec. 2230, 19-28 (2011)

Gescheider, G.A.: Psychophysics: The Fundamentals. Erlbaum, Mahwah (1997)

Guilford, J.P.: A generalized psychophysical law. Psychol. Rev. 39, 73-85 (1932)

Hensher, D.A., Greene, W.H., Chorus, C.G.: Random regret minimization or random utility maximization: an exploratory analysis in the context of automobile fuel choice. J. Adv. Transp. 47, 667-678 (2013)

Hess, S., Stathopoulos, A., Daly, A.: Allowing for heterogeneous decision rules in discrete choice models: an approach and four case studies. Transportation (Amst). 39, 565-591 (2012)

Hess, S., Stathopoulos, A.: Linking response quality to survey engagement: a combined random scale and latent variable approach. J. Choice Model. 7, 1-12 (2013)

Kaplan, S., Prato, C.G.: The application of the random regret minimization model to drivers' choice of crash avoidance maneuvers. Transp. Res. Part F 15(6), 699-709 (2012)

Loomes, G., Sugden, R.: Royal Economic Society Regret theory: an alternative theory of rational choice under uncertainty. Econ. J. 92, 805-824 (1982)

Loomes, G., Sugden, R.: A rationale for preference reversal. Am. Econ. Rev. 73, 428-432 (1983)

Loomes, G., Sugden, R.: Some implications of a more general form of regret theory. J. Econ. Theory. 41, 270-287 (1987)

Prato, C.G.: Expanding the applicability of random regret minimization for route choice analysis. Transportation 41, 351-375 (2014)

Quiggin, J.: Regret theory with general choice sets. J. Risk Uncertain. 8, 153-165 (1994)

Rasouli, S., Timmermans, HJP. Specification of regret-based models of choice behavior: Formal analyses and experimental design based evidence, Transportation, (2015a)

Rasouli, S., Timmermans, H.J.P.: Models of bounded rationality under certainty. In: Rasouli, S., Timmermans, H.J.P. (eds.) Bounded Rational Choice Behavior: Applications in Transport. Emerald Group Publishing, Bingley (2015)

Sheffi, Y.: Urban Transportation Networks. Prentice-Hall, Englewood Cliffs, New Jersey (1985)

Thiene, M., Boeri, M., Chorus, C.G.: Random regret minimization: exploration of a new choice model for environmental and resource economics. Environ. Resour. Econ. 51, 413-429 (2012)

Weber, E.H.: De pulsu, resorptione, auditu et tactu. Annotationes anatomicae et physiologicae. Koehler, Leipzig (1834)

Sunghoon Jang is Ph.D. Candidate of the Urban Planning Group of the Eindhoven University of Technology, the Netherlands. He received his Master degree in Civil and Environmental Engineering from Seoul National University. His research interests include the areas of travel behavior analysis, transportation planning, transportation economics, and intelligent transportation systems. His current work is primarily concerned with the behavioral decision rules based on discrete choice models.

Soora Rasouli is Assistant Professor of the Urban Planning Group of the Eindhoven University of Technology, the Netherlands. She has research interests in activity-based models of travel demand, modeling of choice processes under uncertainty and spatial analysis. She is a member of the editorial board of the International of Transportation, Modern Traffic and Transportation Engineering Research, International Journal of Urban Science, Journal of Traffic and Transportation Planning and JRCS. She is junior career member of the ISCTSC Board of the International Steering Committee for Travel Survey Conferences, and member of the TRB Special Committee on Travel Forecasting Resources. She has acted as guest editor for Environment and Planning B, Travel Behavior and Society, and the Journal of Choice Modelling.

Harry Timmermans is Head of the Urban Planning Group of the Eindhoven University of Technology, the Netherlands. He has research interests in modeling decision-making processes and decision support systems in a variety of application domains, including transportation. He is editor of the Journal of Retailing and Consumer Services, and serves on the board of several other journals in transportation, geography, urban planning, marketing, artificial intelligence and other disciplines. He is Co-chair of the International Association of Travel Behavior Research (IATBR), and member of several scientific committees of the Transportation Research Board. He has also served as member of conference committees in transportation an artificial intelligence. 